\title{
Anesthetic drugs and sustained neuroprotection in acute cerebral ischemia: can we alter clinical outcomes?
}

\author{
Christian Werner, MD, PhD
}

Published online: 25 September 2009

(c) Canadian Anesthesiologists' Society 2009

\begin{abstract}
Anesthetic drugs have been tested for their neuroprotective characteristics for decades because of their potential either to interrupt or to slow the sequence of injurious biochemical and molecular events that ultimately result in irreversible neuronal death. Unfortunately, clinical trials examining the neuroprotective effects of anesthetic agents have failed to translate the experimental evidence. ${ }^{1}$ The purpose of this editorial is to consider the current stage of knowledge regarding anesthetic neuroprotection in the context of identifying future directions for this important field of research.
\end{abstract}

A fundamental issue in establishing the need for perioperative neuroprotection is to identify target populations that will benefit neurologically from specific anesthetic agents. Perioperative cerebral ischemia remains a significant source of morbidity and mortality in cardiac and noncardiac patients alike. ${ }^{2,3}$ For example, the incidence of acute ischemic stroke varies from 0.8 to $8.8 \%$ in patients undergoing peripheral vascular, aortic reconstructive, and cardiac surgery. In patients undergoing colorectal surgery, total hip arthroplasty, and head and neck surgery, the incidence of perioperative stroke varies from 0.3 to $4.8 \%$. Acute neurodegeneration may also present as postoperative neurocognitive decline. It is rather surprising, then, that this important pathogenicity has been disregarded as a target of neuroprotective strategies, yet it clearly deserves a neuroprotective approach.

A second important issue is the need to establish the differential neuroprotective potential of the various general anesthetic agents. The proposed mechanisms of anesthetic

C. Werner, MD, PhD ( $\square)$

Department of Anesthesiology, Johannes Gutenberg-Universität

Mainz, Langenbeckstrasse 1, 55131 Mainz, Germany

e-mail: wernerc@uni-mainz.de neuroprotection include reduction of cerebral metabolism and intracranial pressure, suppression of seizure activity, and lessening of sympathetic discharge. Additionally, anesthetics inhibit synaptic release of excitatory neurotransmitters; they activate inhibitory $\gamma$-aminobutyric acid (GABA) and glycine receptors and slow the intracellular $\mathrm{Ca}^{2+}-$ and $\mathrm{Na}^{+}-$ accumulation. Additionally, anesthetics reduce the generation of reactive free radical species; they increase ischemic tolerance by pre- and post-conditioning, and they also inhibit apoptotic pathways. ${ }^{4}$ Thus, there is considerable evidence that multiple pathways exist by which anesthetic agents have the potential to exert clinically important benefits. But what is the experimental evidence for (sustained) anesthetic neuroprotection? In numerous studies, anesthetic neuroprotection has been confirmed in rodents subjected to focal or incomplete hemispheric ischemia where isoflurane, sevoflurane, desflurane, and xenon, as well as barbiturates and propofol, decreased infarct size and improved motor and cognitive function when given prior to the ischemic challenge. ${ }^{5-9}$ The reduction in ischemic neuronal injury has been shown to be most prominent in study designs comparing "anesthesia" with awake or sedated states and less pronounced when different anesthetics were compared with each other. Neurologic outcome is reproducible only with brief episodes of cerebral ischemia, while longer durations of low-flow states or permanent focal ischemia are resistant to anesthetic neuroprotection. ${ }^{5,10}$ In line with this evidence, anesthetics have no neuroprotective properties in the setting of global cerebral ischemia and when given after the ischemic insult.

It is questionable whether the effects of anesthetics seen in these different ischemia models are permanent. Studies in rats subjected to focal cerebral or incomplete global ischemia have shown that cell death was substantially reduced in the immediate post-ischemic period; however, 
no difference in cortical and subcortical damage was detected within weeks or months. ${ }^{10-12}$ The mechanisms that underlie this progression in neuronal damage are not fully understood, but apoptosis clearly contributes to this effect. Anesthetics economize ischemic energy consumption with consecutive reduction in immediate cell death. Yet the metabolic shift to less energy deprivation is insufficient to entirely restore neuronal integrity, and initiation of apoptosis (an energy-requiring process) will ultimately reverse the initial neuroprotection. This indicates the need for study designs that systematically focus on long-term neuronal viability as the primary endpoint.

Thus, it is apparent that there is indeed evidence of sustained neuroprotection from anesthetic agents in ischemia models of mild injury when the anesthetics are given prior to or during the ischemic challenge and with a short period of post-ischemic observation. However, there is no evidence of such protection in models of permanent (severe) cerebral ischemia when drugs are given after the ischemic insult and with long-term post-ischemic observation.

\section{Is there clinical evidence for anesthetic neuroprotection?}

While experimental data support the preventive neuroprotective effects of anesthetics, the clinical evidence is less convincing. In patients undergoing cardiac surgery with normothermic cardiopulmonary bypass, the infusion of thiopental (total dose during ECC: $39.5 \pm 8.4 \mathrm{mg} \cdot \mathrm{kg}^{-1} i v$ ) was able to reduce postoperative neuropsychological deficits. ${ }^{13}$ Although that study has been challenged for statistical weaknesses, it seems to contrast with the results of other studies, for instance, where barbiturates (infused in comatose patients within the first hour following cardiopulmonary resuscitation) proved ineffective in reducing mortality and neurological deficits in survivors compared with standard ICU treatment being administered to those patients. ${ }^{14}$ These data are consistent with the view that infusing hypnotics prior to focal cerebral rather than global ischemic insults may increase the ischemic tolerance of neurons. Also, barbiturates may be beneficial in patients with severe head injury and refractory intracranial hypertension. This conclusion relates to a series of clinical studies where infusion of barbiturates following brain trauma was effective in reducing intracranial pressure and likely the mortality rate, as long as systemic hemodynamic stability was maintained. ${ }^{15}$ Propofol has been suggested as an alternative to barbiturates, either in patients undergoing cardiac surgery or for sedation following head injury, due to its favourable context-sensitive halftimes after infusions are discontinued. Propofol does not appear to reduce neuropsychological deficits following cardiac valve surgery compared with sufentanil-anesthetized patients; however, when compared with an opioid-based sedative regimen, it shows more effective results in treating elevated intracranial pressure with a similar neurologic outcome following head injury. ${ }^{16,17}$ A multicentre trial in patients undergoing carotid endarterectomy with general or local anesthesia revealed no difference in the occurrence of stroke within 30 days from the time of surgery. ${ }^{18}$ Despite several limitations of that trial, including statistical underpowering, insufficient standardization of the local anesthetic used, and varying levels of sedation in the local anesthetic group, the data re-affirm the view that a single pharmacological approach can hardly affect the multiple simultaneous pathological events in patients with parallel variability of coexisting disease. Thus, no strong clinical evidence remains to support the hypothesis that sustained anesthetic neuroprotection exists.

\section{Why have clinical trials failed to confirm anesthetic neuroprotection in patients?}

The translational failure in the search for clinical neuroprotection emerges from misconceptions of experimental studies. Many studies that presented an invalid hypothesis were based on unrepresentative pre-clinical model systems and failed to properly control important physiological variables, such as brain temperature, plasma glucose concentration, $\mathrm{PaCO}_{2}, \mathrm{pH}$, and blood pressure. Likewise, selection of inadequate endpoints (e.g., surrogate parameters, such as EEG or brain oxygenation, rather than histopathology or post-ischemic neurological testing) and lack of proper statistics in a number of studies led to overinterpretation of data. The above facts can partly explain the failure of clinical investigations examining neuroprotective strategies. With incomplete experimental evidence, there is little chance of improving neurologic outcome from ischemic or traumatic insults. Critical timing of treatment (i.e., administration of anesthetics after the insult) and a study population that is exceptionally sick (e.g., moribund patients following traumatic brain injury) contribute to the unavailability of neuroprotective evidence. Similarly, studies have failed because of heterogenous populations (subgroups), small cohorts, inadequate randomization, and lack of sensitivity of various clinical end-points. ${ }^{19,20}$

\section{Consideration of future research directions}

There is valid experimental research offering some evidence that anesthetic neuroprotection exists following mild ischemic insults. Because translation of that evidence is lacking, based on the factors indicated above, future study 
designs and conduct must be more rigorous and sophisticated. Thus, to help clarify the ultimate clinical potential to protect neurons from perioperative ischemic stress, future studies should include the following features: the selection of homogenous insults, a logical timing of drug application, meticulous control of physiological variables, clear definition of adequate end-points, appropriate statistical methodology, and rigorous statistical analyses with conservative interpretation of data.

\section{Conclusion}

In the last several decades, the pathomechanisms of neuronal damage have been characterized. The diversity of insults (i.e., focal $v s$ global ischemia, hemorrhage, trauma) and the complex and multi-level nature of ischemic injury explain the lack of potent and sustained protection with a monopharmacological approach. Yet most anesthetics have been successfully tested in reproducible and well-controlled animal models of cerebral ischemia or brain trauma. The translational loss appears related to weaknesses in both experimental and clinical study design, and faulty or variable methodologies do not necessarily reflect failure of the concept of anesthetic neuroprotection. Thus, any perspective to alter outcome will relate to the quality and sophistication of pre-clinical and clinical study platforms as formulated in guidelines for experimental research established by the Stroke Therapy Academic and Industry Roundtable (STAIR). ${ }^{21}$ In the meantime, to manage experimental and perioperative cerebral insults, the focus must be on the control of key physiological variables to ensure normotension, normoxia, normocapnia, normothermia, and normoglycemia as potent neuroprotective interventions, which may act independently or synergistically on the long-term neurological patient outcome.

\section{Médicaments anesthésiques et neuroprotection soutenue lors d'ischémie cérébrale aiguë: peut- on modifier les devenirs cliniques?}

Depuis des dizaines d'années, les agents anesthésiques sont testés pour déterminer leurs propriétés neuroprotectrices en raison de leur potentiel d'interruption ou de ralentissement de la séquence d'effets biochimiques ou moléculaires nocifs, lesquels ont pour résultat final une mort neuronale irréversible. Malheureusement, les études cliniques examinant les effets neuroprotecteurs des agents anesthésiques ne sont pas parvenues à traduire les données probantes expérimentales. ${ }^{1}$
L'objet de cet éditorial est de rendre compte du niveau actuel de connaissances en matière de neuroprotection anesthésique, avec pour objectif d'identifier les orientations futures de ce domaine de recherche important.

Une des composantes essentielles lorsqu'on établit le besoin de neuroprotection périopératoire est l'identification des populations cibles qui bénéficieront, sur le plan neurologique, d'agents anesthésiques spécifiques. L'ischémie cérébrale périopératoire demeure une source significative de morbidité et de mortalité, aussi bien chez les patients en chirurgie cardiaque que non cardiaque. ${ }^{2,3}$ Par exemple, l'incidence d'accident ischémique cérébral varie de 0,8 à $8,8 \%$ chez les patients subissant une chirurgie vasculaire périphérique, reconstructive de l'aorte et cardiaque. Chez les patients subissant une chirurgie colorectale, une arthroplastie totale de la hanche et une chirurgie de la tête et du cou, l'incidence d'accident ischémique périopératoire varie entre 0,3 et $4,8 \%$. La neurodégénérescence aiguë peut également se présenter sous forme de déclin neurocognitif postopératoire. Dès lors, il est d'autant plus surprenant que cette pathogénicité ait été négligée comme cible des stratégies neuroprotectrices, alors qu'elle mérite clairement une approche neuroprotectrice.

Le besoin d'établir le potentiel neuroprotecteur différentiel des divers agents anesthésiques généraux constitue un autre enjeu important. Parmi les mécanismes de neuroprotection anesthésique suggérés figurent la réduction du métabolisme cérébral et de la pression intracrânienne, la suppression des crises et la réduction de la décharge sympathique. En outre, les agents anesthésiques inhibent la libération synaptique de neurotransmetteurs excitateurs; ils activent les récepteurs inhibiteurs de l'acide $\gamma$-aminobutyrique (GABA) et de la glycine et ralentissent l'accumulation intracellulaire de $\mathrm{Ca}^{++}$et $\mathrm{Na}^{+}$. De plus, les agents anesthésiques réduisent la génération d'espèces réactives de radicaux libres; ils augmentent la tolérance ischémique en effectuant un conditionnement antérieur et postérieur, et inhibent les voies apoptotiques. ${ }^{4}$ Ainsi, les données probantes soutenant qu'il existe des voies multiples par lesquelles les agents anesthésiques ont le potentiel d'exercer des bienfaits cliniquement importants sont foison. Mais quelles sont les données probantes expérimentales pour la neuroprotection anesthésique (soutenue)? Dans nombre d'études, la neuroprotection anesthésique a été confirmée chez le rat soumis à une ischémie hémisphérique focale ou complète; dans ces études, l'administration d'isoflurane, de sévoflurane, de desflurane et de xénon, ainsi que de barbituriques et de propofol, a réduit la taille de l'infarctus et amélioré les fonctions motrice et cognitive lorsqu'elle avait lieu avant l'exposition à l'ischémie.$^{5-9} \mathrm{Il}$ a été montré que la réduction de la lésion neuronale ischémique était le plus importante dans des modèles d'étude comparant l'état « anesthésié » à des états éveillé ou sous sédation et 
qu'elle était moins prononcée lorsque divers anesthésiques étaient comparés entre eux. Le devenir neurologique peut être reproduit uniquement lors d'épisodes brefs d'ischémie cérébrale, alors que les durées prolongées d'états de faible débit ou d'ischémie focale permanente sont réfractaires à la neuroprotection anesthésique. ${ }^{5,10}$ Conformément à ces données probantes, les anesthésiques n'ont aucune propriété neuroprotectrice dans le contexte d'une ischémie cérébrale globale ou lorsqu'ils sont administrés après la lésion ischémique.

Nous ne savons pas si les effets des agents anesthésiques observés dans ces différents modèles d'ischémie sont permanents. Les études menées sur des rats soumis à une ischémie cérébrale focale ou globale incomplète ont montré que la mort cellulaire était considérablement réduite dans la période suivant immédiatement l'ischémie; toutefois, aucune différence n'a été observée dans les lésions corticales et sous-corticales dans les semaines ou mois suivants. ${ }^{10-12}$ Les mécanismes qui sous-tendent la progression de la lésion neuronale demeurent encore mal compris, mais l'apoptose contribue clairement à cet effet. Les agents anesthésiques économisent la consommation d'énergie ischémique, ce qui provoque une réduction consécutive de la mort cellulaire. Cependant, la conversion métabolique vers une privation énergétique moindre n'est pas suffisante pour restaurer complètement l'intégrité neuronale, et le début de l'apoptose, un processus nécessitant de l'énergie, inversera en fin de compte la neuroprotection initiale. Par conséquent, il est nécessaire de réaliser des études dont le critère principal d'évaluation est systématiquement la viabilité neuronale à long terme.

Dès lors, il est clair qu'il existe des données probantes appuyant l'hypothèse d'une neuroprotection soutenue provoquée par des agents anesthésiques dans les modèles ischémiques de lésion faible et ce, lorsque les anesthésiques sont administrés avant ou pendant le test ischémique et avec une courte période d'observation après l'ischémie. Toutefois, nous n'avons aucune donnée probante soutenant qu'il existe une telle protection dans des modèles d'ischémie cérébrale permanente (grave) lorsque les agents anesthésiques sont administrés après le test ischémique et avec une observation à long terme après l'ischémie.

\section{Y a-t-il des données probantes cliniques appuyant la thèse d'une neuroprotection anesthésique?}

Bien que les données expérimentales soutiennent l'existence d'effets neuroprotecteurs préventifs des anesthésiques, les données probantes cliniques sont moins convaincantes. Une étude rapporte que, chez des patients subissant une chirurgie cardiaque avec une circulation extracorporelle normothermique, une perfusion de thiopental (dosage total pendant le refroidissement d'urgence du cœur : $39,5 \pm 8,4 \mathrm{mg} \cdot \mathrm{kg}^{-1}$ iv) a permis de réduire les déficits neuropsychologiques postopératoires. ${ }^{13}$ Bien que l'étude en question ait été critiquée en raison de ses faiblesses statistiques, elle semble différer des résultats d'autres études dans lesquelles, par exemple, l'administration de barbituriques (perfusés chez des patients comateux durant la première heure suivant la réanimation cardio-respiratoire) s'est avérée inefficace pour réduire la mortalité et les déficits neurologiques chez les survivants, par rapport au traitement standard administré à ces patients à l'unité des soins intensifs. ${ }^{14}$ Ces données sont conformes à l'opinion selon laquelle la perfusion d'agents hypnotiques avant les lésions cérébrales focales plutôt que les lésions ischémiques globales pourrait augmenter la tolérance ischémique des neurones. En outre, les barbituriques pourraient s'avérer bénéfiques pour les patients souffrant d'un traumatisme crânien grave et d'hypertension intracrânienne réfractaire. Cette conclusion est liée à une série d'études cliniques dans lesquelles une perfusion de barbituriques à la suite d'un traumatisme crânien a efficacement réduit la pression intracrânienne et probablement le taux de mortalité, tant que la stabilité hémodynamique systémique était maintenue. ${ }^{15}$ En raison de ses demi-vies d'élimination contextuelles favorables après l'interruption de la perfusion, le propofol a été proposé comme alternative aux barbituriques, soit chez des patients subissant une chirurgie cardiaque ou pour réaliser une sédation après un traumatisme crânien. Le propofol ne semble pas réduire les déficits neuropsychologiques après une chirurgie cardiaque valvulaire par rapport aux patients anesthésiés avec du sufentanil; toutefois, lorsqu'il est comparé à un régime de sédation par opiacés, le propofol a des résultats plus probants pour le traitement d'une pression intracrânienne élevée et résulte en un devenir neurologique semblable après un traumatisme crânien. ${ }^{16,17}$ Une étude multicentrique portant sur des patients subissant une endartériectomie de la carotide sous anesthésie générale ou locale n'a pas révélé de différence dans l'incidence d'infarctus dans les 30 jours suivant la chirurgie. ${ }^{18}$ Cette étude comporte plusieurs faiblesses, notamment le manque de puissance statistique, le peu de standardisation quant à l'anesthésique local utilisé et les niveaux de sédation variés du groupe anesthésique local; toutefois, les résultats réaffirment l'hypothèse selon laquelle une approche pharmacologique unique n'a que peu d'effet sur les nombreux événements pathologiques simultanés qui surviennent chez des patients présentant une variabilité parallèle à cause de maladies coexistantes. Par conséquent, nous n'avons plus aucune donnée probante clinique solide pour soutenir l'hypothèse qu'une neuroprotection anesthésique soutenue existe. 
Pour quelle raison les études cliniques n'ont-elles pas pu confirmer de neuroprotection anesthésique chez des patients?

Le fait que la recherche en matière de neuroprotection clinique ne se soit pas traduite dans la pratique est lié à certaines fausses conceptions présentes dans les études expérimentales. Plusieurs des études ayant présenté une hypothèse invalide étaient fondées sur des systèmes de modèles précliniques non représentatifs et ne sont pas parvenues à contrôler de façon adéquate certaines variables physiologiques essentielles comme la température du cerveau, le taux de glycémie veineuse, la $\mathrm{PaCO}_{2}$, le $\mathrm{pH}$ et la pression artérielle. De même, le choix de critères inappropriés (par ex., des paramètres de substitution tels que l'EEG ou l'oxygénation cérébrale plutôt que l'histopathologie ou les tests neurologiques post-ischémie) et le manque de statistiques adaptées ont causé, dans plusieurs études, une sur-interprétation des données. Ceci pourrait expliquer en partie l'échec des études cliniques portant sur les stratégies de neuroprotection. Si les données probantes expérimentales s'avèrent incomplètes, il sera difficile d'améliorer le devenir neurologique causé par une lésion ischémique ou traumatique. Le moment critique d'administration du traitement (c.-à-d. l'administration d'agents anesthésiques après la lésion) et une population de patients particulièrement malade (par ex., patients moribonds à la suite d'un traumatisme crânien) sont d'autres facteurs contribuant au manque de données appuyant l'effet neuroprotecteur des anesthésiques. De même, certaines études ont échoué en raison de populations hétérogènes (sousgroupes), de cohortes trop petites, d'une randomisation inadaptée et du manque de sensibilité de certains critères d'évaluation cliniques. ${ }^{19,20}$

\section{Orientations futures pour la recherche: quelques remarques}

Il existe certaines recherches expérimentales valides présentant des données probantes soutenant qu'il existe une neuroprotection anesthésique après des lésions ischémiques légères. Étant donné que ces données n'ont pas pu être traduites dans la recherche clinique, en raison des facteurs mentionnés ci-dessus, les méthodologies des recherches futures devront être à la fois plus rigoureuses et plus sophistiquées. Ainsi, afin d'aider à déterminer le véritable potentiel clinique des agents anesthésiques à protéger les neurones du stress ischémique périopératoire, les études devront à l'avenir être dotées des propriétés suivantes : la sélection de lésions homogènes, un choix logique du moment opportun d'administration du médicament, un contrôle méticuleux des variables physiologiques, une définition claire de critères d'évaluation adaptés, une méthodologie statistique adéquate et des analyses statistiques rigoureuses accompagnées d'une interprétation conservatrice des données.

\section{Conclusion}

Au cours des dernières décennies, les pathomécanismes des lésions neuronales ont été décrits. La diversité des lésions (c.-à-d. ischémie focale vs globale, hémorragie, traumatisme) et la nature complexe et multidimensionnelle de la lésion ischémique expliquent le manque de protection puissante et soutenue pouvant être obtenue avec une approche mono-pharmacologique. Cependant, la plupart des anesthésiques se sont avérés efficaces dans des modèles animaux reproductibles et bien contrôlés d'ischémie cérébrale ou de traumatisme crânien. Il semble que l'échec de traduction de ces résultats dans la pratique clinique soit due à des faiblesses au niveau de la conception des études expérimentales aussi bien que cliniques; en outre, les méthodologies erronées ou variables ne reflètent pas forcément l'invalidité de l'idée de neuroprotection anesthésique en tant que telle. Dès lors, si l'on souhaite influencer les devenirs, la qualité et la sophistication des plates-formes d'études précliniques et cliniques devront être améliorées, en se référant aux directives en matière de recherche expérimentale établies par la STAIR (Stroke Therapy Academic and Industry Roundtable). ${ }^{21}$ En attendant, lors de la prise en charge des lésions cérébrales expérimentales et périopératoires, il est essentiel de mettre l'emphase sur la surveillance des variables physiologiques clés afin de garantir une tension artérielle normale, la normoxie, la normocapnie, la normothermie, et la normoglycémie, car ces variables constituent des interventions neuroprotectrices puissantes qui pourraient avoir un effet indépendant ou en synergie sur le devenir neurologique à long terme du patient.

Competing interests None declared.

\section{References}

1. Ginsberg M. Neuroprotection for ischemic stroke: past, present and future. Neuropharmacology 2008; 55: 363-89.

2. Selim M. Perioperative stroke. New Engl J Med 2007; 356: 706-13.

3. Bateman BT, Schumacher HC, Wang S, Shaefi S, Berman MF. Perioperative acute ischemic stroke in noncardiac and nonvascular surgery: incidence, risk factors, and outcome. Anesthesiology 2009; 110: 231-8.

4. Kawaguchi M, Furuya H, Patel PM. Neuroprotective effects of anesthetic agents. J Anesth 2005; 19: 150-6.

5. Engelhard K, Werner C, Eberspacher E, et al. Sevoflurane and propofol influence the expression of apoptosis-regulating proteins after cerebral ischaemia and reperfusion in rats. Eur $\mathbf{J}$ Anaesthesiol 2004; 21: 530-7. 
6. Engelhard K, Werner C, Reeker W, et al. Desflurane and isoflurane improve neurological outcome after incomplete cerebral ischaemia in rats. Br J Anaesth 1999; 83: 415-21.

7. Sakai H, Sheng H, Yates RB, Ishida K, Pearlstein RD, Warner $D S$. Isoflurane provides long-term protection against focal cerebral ischemia in the rat. Anesthesiology 2007; 106: 92-9.

8. Homi HM, Yokoo N, Ma D, et al. The neuroprotective effect of xenon administration during transient middle cerebral artery occlusion in mice. Anesthesiology 2003; 99: 876-81.

9. Warner DS, Takaoka S, Wu B, et al. Electroencephalographic burst suppression is not required to elicit maximal neuroprotection from pentobarbital in a rat model of focal cerebral ischemia. Anesthesiology 1996; 84: 1475-84.

10. Bayona NA, Gelb AW, Jiang Z, Wilson JX, Urquhart BL, Cechetto DF. Propofol neuroprotection in cerebral ischemia and its effects on low-molecular-weight antioxidants and skilled motor tasks. Anesthesiology 2004; 100: 1151-9.

11. Kawaguchi M, Kimbro JR, Drummond JC, Cole DJ, Kelly PJ, Patel PM. Isoflurane delays but does not prevent cerebral infarction in rats subjected of focal ischemia. Anesthesiology 2000; 92: 1335-42.

12. Elsersy H, Sheng H, Lynch JR, Moldovan M, Pearlstein RD, Warner DS. Effects of isoflurane versus fentanyl-nitrous oxide anesthesia on long-term outcome from severe forebrain ischemia in the rat. Anesthesiology 2004; 100: 1160-6.

13. Nussmeier NA, Arlund C, Slogoff S. Neuropsychiatric complications after cardiopulmonary bypass: cerebral protection by a barbiturate. Anesthesiology 1986; 64: 165-70.
14. Anonymous. Randomized clinical study of thiopental loading in comatose survivors of cardiac arrest. Brain Resuscitation Clinical Trial I Study Group. N Engl J Med 1986; 314: 397-403

15. Bullock MR, Povlishock JT. Guidelines for the management of severe traumatic brain injury. Editor's commentary. J Neurotrauma 2007; 24: S71-6.

16. Kelly DF, Goodale DB, Williams J, et al. Propofol in the treatment of moderate and severe head injury: a randomized, prospective double-blinded pilot trial. J Neurosurg 1999; 90: $1042-52$.

17. Roach GW, Newman MF, Murkin JM, et al. Ineffectiveness of burst suppression therapy in mitigating perioperative cerebrovascular dysfunction. Multicenter Study of Perioperative Ischemia (McSPI) Research Group. Anesthesiology 1999; 90: 1255-64.

18. GALA Trial Collaborative Group, Lewis SC, Warlow CP, Bodenham AR, et al. General anaesthesia versus local anaesthesia for carotid surgery (GALA): a multicentre, randomised controlled trial. Lancet 2008; 372: 2132-42.

19. Dirnagl $U$. Bench to bedside: the quest for quality in experimental stroke research. J Cereb Blood Flow Metab 2006; 26: 1465-78.

20. Hoyte L, Kaur J, Buchan AM. Lost in translation: taking neuroprotection from animal models to clinical trials. Exp Neurol 2004; 188: 200-4.

21. Macleod MR, Fisher M, O'Collins V, et al. Good laboratory practice: preventing introduction of bias at the bench. Stroke 2009; 40: e50-2. 